\title{
"Le soldat n'a pas fui, il est parti chercher de la force" : explorer les imaginaires migratoires à l'aune des carrières artistiques dans le rap au Sénégal
}

"The Soldier didn't Run Away, He Went Looking for Strength": Exploring

Migration Imaginaries through Artistic Careers in Rap in Senegal

«El soldado no escapo, se fue a buscar fuerza»: explorar los imaginarios

migratorios a través de las carreras artísticas en el rap en Senegal

\section{Cécile Navarro}

\section{(2) OpenEdition Journals}

Édition électronique

URL : https://journals.openedition.org/remi/12858

DOI : $10.4000 /$ remi. 12858

ISSN : $1777-5418$

Traduction(s) :

"The Soldier didn't Run Away, He Went Looking for Strength": Exploring Migration Imaginaries through Artistic Careers in Rap in Senegal - URL : https://journals.openedition.org/remi/13166 [en]

Éditeur

Université de Poitiers

Édition imprimée

Date de publication : 1 octobre 2019

Pagination : 149-169

ISBN : 979-10-90426-64-1

ISSN : 0765-0752

Référence électronique

Cécile Navarro, «"Le soldat n'a pas fui, il est parti chercher de la force" : explorer les imaginaires migratoires à l'aune des carrières artistiques dans le rap au Sénégal », Revue européenne des migrations internationales [En ligne], vol. 35 - $n^{\circ} 1$ et 2 | 2019, mis en ligne le 01 janvier 2021, consulté le 16 avril 2022. URL : http://journals.openedition.org/remi/12858; DOI : https://doi.org/10.4000/remi. 12858 


\title{
" Le soldat n'a pas fui, il est parti chercher de la force "): explorer les imaginaires migratoires à l'aune des carrières artistiques dans le rap au Sénégal
}

\author{
Cécile Navarro ${ }^{1}$
}

Encore largement inexplorée (Martiniello, 2015), I'étude des expressions artistiques en migration a permis d'affirmer le rôle important de l'art dans la vie quotidienne des migrants, encore majoritairement perçus sous la figure du " travailleur ". Martiniello et al. (2009) consacrent notamment un numéro de la Revue Européenne des Migrations Internationales aux formes théâtrales, musicales, poétiques, littéraires et plastiques qui sont l'œuvre de personnes ou de milieux artistiques en circulation. Par ailleurs, le regard projeté sur des artistes, acteurs très mobiles sans pour autant faire partie d'une élite socio-économique, a apporté un nouvel éclairage dans l'analyse des phénomènes transnationaux, à l'image du numéro spécial dirigé par Kiwan et Meinhof paru dans la revue Music and Arts in Action. Les créations musicales, en tant que moyen d'expression des expériences vécues par leurs auteurs et porteuses de représentations, nous renseignent aussi sur la production $d^{\prime}$ " imaginaires migratoires ", à même de valoriser la voix des migrants eux-mêmes (Canut et Sow, 2014).

Cet article tente de combiner ces différentes approches de l'art en situation de migration et de mobilité par la mise en dialogue entre pratiques, perceptions et représentations imaginaires du mouvement (Ortar et al., 2018), à partir de données issues d'un terrain ethnographique "tout-terrain "² (Meyer et al., 2017) auprès de personnes impliquées dans la production de la musique rap

\footnotetext{
1 Post-doctorante et Chercheuse affiliée à I'Université de Lausanne, Haute École de Suisse Occidentale Valais, Institut Santé, Chemin de I'Agasse 5, 1950 Sion, Suisse, ; cecile.navarro@hevs.ch

La citation du titre de I'article est une traduction de la version originale en wolof " Jambaar dawul dolé ladoon wuti " (Daara J Family, 2016).

2 Par " tout terrain ", les auteurs de ce dossier de SociologieS désignent le terrain ethnographique qui s'effectue au-delà d'une co-présence prolongée sur un lieu unique. L'ethnographe est par exemple de plus en plus souvent amené à suivre un phénomène qui traverse plusieurs lieux. Ma pratique du " tout terrain " s'est effectué par la fréquentation de différents lieux de production et d'écoute de la musique rap sénégalaise, y compris sur Internet, ainsi que par l'organisation d'une co-présence répétée avec les artistes dans différents lieux du terrain dakarois et à l'étranger, au cours d'expériences de mobilité.
} 
au Sénégal (artistes, producteurs, acteurs culturels). M'inspirant du " mobility turn " (Sheller et Urry, 2006), j'ai envisagé dans ma recherche doctorale (Navarro, 2019) ${ }^{3}$ les régimes de mobilité (Glick Schiller et Salazar, 2013) à I'œuvre dans la construction des carrières artistiques : si la mobilité peut donner lieu à de nouveaux modes de consécration et de réussite, son impossibilité peut aussi être productrice d'exclusion, de marginalisation et d'inégalités sociales. Par carrière artistique, il est entendu, avec Becker (1985), un processus de changement de statut et de position, qui évolue dans le temps et qui tend vers la réussite. Force est de constater, au sujet des carrières artistiques, que la mobilité de l'artiste est souvent significative d'un changement de position et de statut (Le Menestrel, 2012) ainsi que d'une accession, parfois imaginaire, à la réussite.

Dans cet article, je souhaite plus précisément montrer comment ces régimes de mobilité apparaissent à l'aune d'imaginaires migratoires diffusés par des chansons qui sont le fait d'interprètes qui ont, par eux-mêmes, fait l'expérience de la mobilité. Pour ce faire, il sera question d'envisager les imaginaires migratoires de certaines chansons de rap, non seulement à l'aide de leurs paroles et de différents supports artistiques (les clips vidéos notamment), mais aussi à partir de ce qu'elles nous révèlent de la façon dont ses interprètes mettent en scène leurs carrières artistiques comme accession à la réussite par la mobilité. La restitution des carrières artistiques est effectuée au moyen d'entretiens avec les artistes, complétés par des sources secondaires (site web de l'artiste, articles journalistiques). Avant d'entreprendre cette analyse à partir des exemples de deux groupes de rap sénégalais, Daara J Family et Wagëblë, il sera question d'envisager la façon dont le genre musical rap, et le rap au Sénégal plus précisément, sont des exemples particulièrement pertinents pour mener à bien cette démarche d'analyse.

\section{Le rap, représentation fidèle d'une parole migrante?}

Fin septembre 2018, le mouvement de la jeunesse suisse romande (MJSR) diffuse sur la plateforme de vidéos YouTube le clip "Je viens de loin ", réalisé lors d'un " camp hip-hop " destiné à " produire, réaliser et promouvoir l'art et la musique urbaine dans un objectif artistique et social $"{ }^{4}$. Quinze mineurs non accompagnés provenant de divers pays s'y expriment sur leurs parcours migratoires. Cette utilisation privilégiée du genre musical rap pour témoigner de l'expérience migratoire n'est pas nouvelle, et elle n'est pas non plus dénuée d'éléments problématiques. Au-delà de sa facilité d'accès à des musiciens non professionnels (Traïni, 2008), en quoi le rap serait-il un genre musical plus à même de rendre compte d'un vécu de la migration?

Aux États-Unis, berceau du rap, le genre musical s'est longtemps affirmé comme un mode d'expression afro-américain, dernier héritier des pratiques musicales des populations africaines esclaves. II est donc d'abord censé narrer les conditions de vie des populations les plus marginalisées du rêve américain,

3 Cette recherche, menée entre 2014 et 2018, a été menée avec le soutien du Fonds National Suisse pour la recherche scientifique (FNS), sous la direction de Monika Salzbrunn (ISSRUniversité de Lausanne) et la co-direction de Heidrun Friese (TU Chemnitz).

4 https://www.20min.ch/ro/musique/news/story/lls-racontent-leur-migration-dans-un-clipde-rap-12928596 
issues de quartiers touchés par la misère, la violence et la discrimination (Rose, 1994). En France, deuxième marché de production et de consommation du rap, le genre musical a immédiatement été saisi comme un " phénomène culturel propre aux grands ensembles périphériques " (Milliot, 2006 : 180). Cette association du rap en France à la " banlieue " (Hammou, 2016), et donc à l'" immigration ", en posant les rappeurs comme représentatifs de " jeunes de banlieue " d'origine immigrée, et leurs paroles comme expression directe du vécu de ces quartiers, a inscrit le rap comme signe visible du "problème des banlieues ", territoires qui "symbolisent la concentration des phénomènes de l'exclusion et cristallisent les peurs face à l'insécurité "(Avenel, 2009 : 36). D'autre part, le rattachement des rappeurs eux-mêmes à certains territoires (Forman, 2000), à des vécus migratoires - comme le " rap de fils d'immigré " identifié par Clément (2015) - ou encore à des vécus criminels - qui apparaît par exemple dans la reprise récurrente du terme thug, "voyou " (Jeffries, 2011) -, vient confirmer ces catégorisations. En reprenant des assignations dépréciatives pour se les approprier, les artistes de rap contribuent paradoxalement à renforcer ces assignations et à reproduire les stéréotypes pesant sur cette pratique artistique ainsi que sur les territoires dont ils se revendiquent comme les " représentants " (Guillard, 2012). Tel que résumé par Bazin (2001), le rap est irrémédiablement associé au " ghetto " et à l'immigration.

Dans les contextes africains, le rap est en revanche majoritairement perçu comme le mode d'expression d'une rupture générationnelle (Diouf, 2002). L'étude de la pratique du rap en contexte africain déplace ainsi le regard des immigrés vers une autre catégorie sociale, les hommes jeunes, plus susceptibles d'entreprendre l' " aventure " migratoire. Les recherches qui se sont intéressées à la musique rap dans ses contextes posent ainsi la question du rôle de la musique dans la diffusion des représentations de la migration. Chez Souiah (2011), le mezoued, le rap tunisien et les chants de stade sont choisis précisément parce que, par leurs auditeurs, ces genres musicaux diffusent des représentations de la migration auprès des populations qui migrent le plus : de jeunes hommes urbains et marginalisés. Chez Salzbrunn et al. (2015), la musique populaire des harragas tunisiens, dont le rap, est explorée comme " un révélateur de l'imaginaire migratoire et de dynamiques sociales profondes " et s'articule autour de quatre thèmes : les désirs migratoires, les dangers de la traversée, la souffrance de l'exilé et des siens, l'acceptation de la volonté divine.

Au Sénégal, à la suite de l'augmentation des flux de départs en pirogue à destination des îles Canaries dans les années 2006-2007, connues sous le slogan en wolof de Barça walla Barsax ("Barcelone ou l'au-delà ") (Willems, 2008), exprimant la détermination de migrants à rejoindre l'Espagne, et donc l'Europe, au péril de leurs propres vies, de nombreux artistes de rap sénégalais diffusent au contraire des chansons dans le but de dissuader les " migrations clandestines ${ }^{5}$ " (Moulard, 2014 ; Rofheart, 2010). Comment expliquer ses représentations majoritairement négatives de la migration au sein d'un genre musical dont les auditeurs sont pourtant de façon importante eux-mêmes concernés par la migration non documentée et que les rappeurs auraient vocation à " représenter " ?

5 C'est le terme utilisé dans les créations artistiques envisagées. Je préfère en revanche utiliser le terme de "migrations non documentées " : c'est l'absence de documents et non I'acte illégal qui désigne cette migration. 
La prise en compte des conventions propres à cette pratique artistique, notamment concernant le statut donné à la parole du rappeur au Sénégal, permet de résoudre cet apparent paradoxe. La capacité pour le rappeur de " représenter ", à savoir de rendre explicite ses liens avec des territoires, est en effet ce qui lui procure la légitimité pour parler au nom de ce territoire et des populations qui les habitent. Moulard-Kouka (2009) a, dans le cas du rap sénégalais, relevé l'apparition de nouvelles délimitations territoriales par l'émergence $d$ 'une nouvelle génération d'artistes se réclamant d'une appartenance territoriale à la " banlieue " dakaroise ${ }^{6}$, par opposition aux quartiers résidentiels du centre-ville dont étaient originaires les groupes pionniers du rap sénégalais. Cette appartenance à la " banlieue " leur permettait alors, selon eux, de parler des problèmes qui touchaient la population sénégalaise et que leur musique, décrite comme "hardcore ", sous-entendu sans concession, se devait d'exprimer. La manière dont l'interprète revendique être le " représentant " d'un groupe est donc sujet, dans le rap, à des modes de consécration artistique basés sur des valeurs d'authenticité, de crédibilité et de légitimité.

Moulard (2014) souligne en revanche que, si les thèmes abordés dans les chansons de rap sur la migration prennent pour objet les difficiles conditions de vie des sans-papiers ou encore le péril des traversées en bateaux, leurs interprètes bénéficient, quant à eux, de conditions de mobilité privilégiées. L'artiste de rap sénégalais Keyti, auteur de la chanson "Eldorado " qui traite du vécu d'un migrant sénégalais en Europe, a mis en évidence, au cours de notre entretien, cet écart entre les expériences racontées dans les chansons et l'expérience propre des interprètes :

"En fait quand j'écrivais ce texte, je n'avais même jamais été en France, mais bon, après c'est ça le pouvoir du texte de rap quoi. De vraiment pouvoir s'imaginer des choses, créer des réalités. " (Entretien, Dakar, 2014)

II m’explique avoir été inspiré par une conversation téléphonique avec un cousin vivant en France. Le rappeur peut donc se saisir de l'expérience migratoire à partir d'histoires vécues par d'autres. Pour Hammou (2005), la véracité du récit de rap tient ainsi davantage d'un " travail artistique de véridiction " que d'un " travail de véridiction ": il s'agirait de jouer, au travers de la parole artistique, sur une parole de vérité plutôt que de reproduire une parole de réalité. Autrement dit, le rap peut raconter une fiction tant qu'elle semble vraie, une démarche artistique dans laquelle s'enchâsse la violence verbale comme seule capable de porter une vérité que personne ne veut entendre. Chez Keyti, ce " travail artistique de véridiction " s'effectue par la mise en scène d'une parole migrante qui ose dire la " vérité " sur les conditions de vie vécues par les migrants dans les pays d'accueil, à l'inverse des " vrais " migrants qui, eux, entretiendraient le fantasme de la vie en Europe :

6 Le terme est utilisé pour désigner les quartiers à la périphérie de la ville de Dakar ainsi que les trois villes faisant partie de la région de Dakar (Pikine, Guédiwaye, Rufisque). Ces espaces, densément peuplés, concentrent des problèmes d'aménagements urbains architecture anarchique, manque d'infrastructures, fréquence des inondations - qui sont, aux yeux des rappeurs, le signe du manque d'intérêt des décideurs politiques pour ces espaces et les populations qui les habitent. 
"Au Sénégal c'est dur, mais ici ce n'est pas aussi facile qu'ils nous font croire Pour le savoir, tu dois venir voir par toi-même nos conditions de vie Parce que tu ne peux pas te l'imaginer

Parce que ce qu'ils te font voir à la télévision n'est rien en comparaison à ce qu'ils ne te montrent pas, ils te cachent presque la vérité Mais les gars quand ils viennent en vacances vendent du rêve

Et, comme tu disais, du début à la fin ils font les joyeux Ensuite, comment convaincre les gens qu'en Europe c'est difficile comme partout " (Keyti, 2003, traduction du wolof au français par Keyti)

"Représenter " n'équivaut donc pas ici à " être un exemple de ", mais plutôt à " agir au nom de quelqu'un ». Comme le résume l'historien sénégalais Benga (2002 : 302) au sujet des artistes de rap au Sénégal :

"Aux yeux des artistes rap, la prise de parole est le fait d'une élite, de ceux qui ont le pouvoir de parler et la capacité de le faire. C'est pour cela que les artistes rap, lorsqu'ils s'adressent aux jeunes, parlent aussi à leur place" ".

La représentation par les rappeurs des " jeunes " s'exerce ainsi par le fait de "parler " en leur nom, davantage que par le fait de refléter leurs comportements. Tout en se présentant comme " jeunes ", les rappeurs n'en agissent pas moins comme de "grands frères ", qui conseillent leurs auditeurs sur les comportements à adopter. De façon analogue, tout en correspondant aux profils des candidats à la migration, les rappeurs utilisent leur moyen d'expression pour mettre en lumière le risque pris par les migrants au cours de leur traversée " clandestine ".

Par conséquent, ces chansons de rap ne doivent pas être considérées uniquement comme le lieu d'expression d'un imaginaire migratoire préexistant, mais aussi comme le lieu d'un discours tenu par des artistes qui font valoir leurs positionnements en accord avec une pratique artistique régie par des conventions (Becker, 2008), notamment au sujet du rôle ambivalent de la mobilité dans les carrières artistiques des artistes de rap sénégalais.

\section{Saisir le rap sénégalais au prisme de la mobilité}

À l'aune de la célébration des trente ans du "rap galsen ", nom donné par ses protagonistes au rap sénégalais, décrétée par la structure de promotion des cultures urbaines Africulturban, nombreux sont ceux qui ont développé leurs carrières artistiques grâce à la mobilité. Le président d'Africulturban, Matador, a créé cette structure à la suite de tournées réalisées en Belgique avec son précédent groupe, le Wa BMG 44. Depuis la création de la structure en 2006, la réputation de son festival international, le Festa $2 \mathrm{H}$, n'a cessé de s'accroître grâce aux partenariats que son directeur, Amadou Fall Ba, tisse de par le monde pour rencontrer des artistes et financer leur venue au Sénégal. Les mobilités croissantes des artistes, dans le cadre de tournées, de collaborations artistiques ou d'invitations en tout genre, ont aussi facilité la multiplication de home studio à Dakar grâce au matériel financé et acquis par les artistes au cours de leurs voyages. Les aspirations à l' « exportation » de nombreux acteurs du rap, constatées lors de ma recherche, correspondent aussi avec la façon dont la migration, au Sénégal, est conçue, par les candidats à la migration, comme un moyen de 
réalisation du soi (Fouquet, 2007), un moyen d'échapper aux contraintes sociales (Mbodji, 2008), ou encore un moyen d'émancipation et d'affirmation de soi (Timera, 2001).

Ces pratiques et imaginaires de l'(im)mobilité dans le rap sénégalais s'inscrivent dans la longue durée du processus de développement de ce genre musical au Sénégal et des connexions tissées, au travers de cette pratique artistique, avec le reste du monde. Selon Charry (2012), c'est aux Sénégalais qui ont émigré, en France et aux États-Unis notamment, et qui retournent au pays pour les vacances, que l'on doit l'arrivée du hip-hop au Sénégal. Certains jeunes, notamment parmi des élites dakaroises sensibles aux tendances provenant de l'étranger, commencent par imiter les pas de danse des bboys ${ }^{7}$ et à en adopter les codes vestimentaires (Herson, 2011). Pour Martin (2004), les États-Unis sont la première influence des jeunes des villes africaines:

"C'est parce que les jeunes urbains voulaient rompre avec un patrimoine rural jugé bien trop encombrant, et avec des formes européennes trop associées à l'oppression coloniale qu'ils ont choisi l'Amérique. Elle seule, dans sa diversité, pouvait donner sens à leurs expériences en manifestant de manière éclatante leur capacité de création, donc de fabrication de modernité autonome pleine de la promesse d'un avenir indépendant. "

Les premiers artistes de rap revendiquent progressivement leur expression musicale comme étant authentiquement sénégalaise, que ce soit par l'expression d'appartenances territoriales, l'usage de langues nationales (notamment du wolof) au détriment du français et de I'anglais, ainsi que par leurs prises de parole sur l'actualité politique et sociale. Selon I'historien sénégalais Diouf (2002), les premiers rappeurs sont apparus au lendemain des élections présidentielles de 1988, à la suite desquelles le président Abdou Diouf est réélu, malgré un antagonisme croissant d'une frange de la jeunesse. Le rap aurait été le mode d'expression privilégié d'une jeunesse déçue par les limites du modèle démocratique sénégalais, mais aussi d'une jeunesse désœuvrée, cherchant à s'occuper pendant I'année blanche qui suivit l'élection. Au milieu des années 1990, des artistes personnifient, aux côtés de sportifs, le mouvement Bul Faale qui tire son nom d'une chanson du groupe de rap Positive Black Soul. Ce mouvement, qui se traduit par " un processus d'individualisation " de la jeunesse sénégalaise et à une remise en cause des valeurs sociales des aînés (Havard, 2001), semble porter ses fruits lors des élections présidentielles de 2000, qui se soldent par la victoire de I'opposant à Abdou Diouf, Abdoulaye Wade, dont la campagne s'est articulée sur le thème du sopi (" changement " en wolof). Par l'appel à l'inscription des nouveaux votants sur les listes électorales puis par l'appel au vote, les artistes du Bul Faale apparaissent comme des acteurs clés de la démocratie au Sénégal, un pays dont l'alternance présidentielle pacifique apparaît alors comme une exception dans la région. À cette reconnaissance locale s'ajoute la reconnaissance internationale de groupes comme le Positive Black Soul, Pee Froiss et Daara J, qui signent des contrats avec de grandes majors internationales aux débuts des années 2000. Cependant, cet " âge d'or " ne dure pas. En premier lieu, ces groupes rompent rapidement leurs contrats et se séparent, entraînant une désillusion vis-à-vis de la possibilité pour un groupe de rap sénégalais de s'« exporter ". D'autres groupes, comme le Rap'adio et le Wa BMG 44, reconnus

7 Premiers adeptes de la danse hip-hop (Bazin, 2001). 
pour leur implication lors des élections de 2000 ainsi que par leur critique sans ménagement du gouvernement, se séparent après le départ de certains de leurs membres à l'étranger. Daddy Bibson, ancien membre des groupes Rap'adio et Pee froiss, s'installe en Suède, puis aux États-Unis. Au milieu des années 2000, que ce soit par la voie documentée ou non, les artistes de rap sont massivement concernés par les départs massifs : le départ à l'étranger apparaît ainsi comme une des causes majeures de séparation des groupes de rap. C'est ce qui pousse notamment le rappeur Maxi Krezy à écrire la chanson "Départ volontaire ", que certains ont interprétée comme étant dirigée spécifiquement contre Daddy Bibson, un ami d'enfance. II y raconte la façon dont il projetait avec l'un de ses amis de conquérir l'Europe grâce à sa musique, en signant un contrat avec une maison de disques, ce qui leur permettrait de gagner de l'argent puis de se marier. Mais I'ami en question ne rentre pas au délai fixé par son visa Schengen. Le troisième couplet de la chanson est assez explicite sur le sort que l'interprète réserve aux rappeurs qui ont quitté le Sénégal :

"Depuis dix ans on espère gagner de l'argent Dans le rap, chacun se demande quand le morceau percera en France Allemagne Espagne

New York Tokyo on attend on ne s'est jamais arrêté yo On emmerde tout rappeur qui a fait un départ volontaire Voyager au nom du Hip-hop une fois arrivé en Europe tu te bazardes croyant que Dieu ferme tous les accès

Négro tu penses que si tout le monde faisait comme toi que deviendra l'avenir du hip-hop sénégalais

Les jeunes qui vous suivent et vous motivent, qui va les encourager? Dans tous les cas, ceux qui sont restés et moi vous envoyons cette lettre Si toutefois vous avez souillé vos mains en torchant le cul des enfants blancs Ne touchez plus à nos micros vous êtes des chats qui voulaient jouer aux malins pour devenir des lions, on n'est plus potes L'aventure doit être sincère et vu que vous avez pris une autre route (...), on vous libère "

(Maxi Krezy, 2003, traduction du wolof au français par El Hassan Diouf, journaliste à Rapdjolof.com)

Cette définition de l'artiste de rap comme celui qui " encourage les jeunes" et lutte pour l'avenir de son pays conduit à une conception de la migration comme un acte lâche, par lequel le rappeur abandonne le rôle qui est le sien dans la société sénégalaise et acte une rupture avec les autres rappeurs restés au Sénégal. Par conséquent, comme le fait remarquer un artiste de rap qui réside aujourd'hui à Paris avec sa femme espagnole, les désirs de migrer des artistes, bien que très présents, ne sont que rarement avoués:

"Au Sénégal 90 \% des artistes qui font du hip-hop, qui font du rap, bon même s'ils le disent pas, c'est vrai que voilà quoi, c'est pour voyager."

(Entretien, Clichy-sous-Bois, 2017)

L'analyse de l'imaginaire migratoire dans les chansons de rap au Sénégal ne peut donc faire l'économie des rapports ambivalents de ces artistes avec la mobilité : source de reconnaissance artistique, la mobilité de l'artiste est présentée comme désirable tant qu'elle n'aboutit pas à un départ définitif, qui remet alors en cause la réputation de l'artiste comme attaché au devenir de son 
pays. Les représentations de la migration telles qu'elles apparaissent dans les chansons doivent alors être mises en perspective en regard de la carrière artistique et des expériences de mobilité de leurs interprètes.

La pertinence de cette mise en perspective se révèle à la lumière d'une analyse comparée entre deux groupes : un groupe internationalement reconnu, mais qui n'a jamais quitté le Sénégal (Daara J Family) et un groupe dont les membres sont partis à l'étranger, mais qui continue à faire de la musique à destination, au moins en partie, du public sénégalais (Wagëblë).

\section{Daara J Family, « le Sénégal est ton abri "}

Le groupe est composé de deux amis de lycée, Ndongo D et Faada Freddy, dont l'oncle, qui habite en France, ramène fréquemment de la musique et des cassettes dans ses valises. C'est par ce moyen que les deux amis découvrent le hip-hop au travers des enregistrements des émissions de Dj Sidney, diffusées en 1984 sur la chaîne de télévision française TF1. Ils rencontrent le troisième membre du groupe, Alaji-man, au cours d'une soirée rap organisée au Métropolis ${ }^{8}$ en 1993 et forment le groupe Daara J en 1994. Une année plus tard, le groupe publie une première cassette éponyme, qui connaît un grand succès au Sénégal avec 15000 exemplaires vendus. Aux côtés de groupes comme Jant bi et Sunu Flavor, le groupe Daara $\mathrm{J}$ développe surtout des thèmes amoureux dans leur musique. Cependant, leur originalité réside dans l'expression musicale de leurs croyances religieuses, ce qui leur permet de conquérir un large public. Le terme Daara ji sert d'ailleurs au Sénégal à désigner l'école coranique.

C'est en France que le groupe se fait " découvrir " par un producteur anglais, Carlton Cigler, qui remixe les chansons de leur cassette pour la sortie de leur premier album international, publié sous le label français Déclic Communication en 1997. C'est aussi sous ce label que sort leur album suivant, Xalima une année plus tard. Cet album leur permet d'effectuer une tournée européenne (France, Belgique, Espagne et Allemagne) durant l'année 2000.

Désirant accéder à une reconnaissance internationale au-delà du marché francophone, les membres du groupe rompent leur contrat avec leur label pour signer sur la filiale anglaise de la major du disque BMG Entertainment (fusionnée avec Sony BMG pour devenir Sony Music en 2005). Le groupe publie leur troisième album international Boomerang en 2003 sur lequel ils enregistrent des collaborations avec plusieurs artistes de renommée internationale, dont la chanteuse malienne Rokia Traoré et le rappeur français Diziz La Peste. Le groupe entame une tournée française au cours de laquelle ils font la première partie des Rita Missouko au Grand Rex à Paris. Après des années de concerts à l'étranger, ainsi que l'obtention du prestigieux prix du BBC World Music Awards pour la catégorie " meilleur artiste africain " en 2004, la carrière internationale du groupe ne décolle pas. En 2007, Alaji-Man quitte le groupe et les deux membres restants se rebaptisent sous le nom de Daara J Family.

8 Ancienne boîte de nuit du centre-ville de la capitale, transformée en restaurant. 
En 2010, le groupe publie son nouvel album School of life sous le label britannique Wrasse Records, spécialisé en musiques du monde. Selon Ndongo D, en les classant dans la rubrique " musique du monde ", le label ne leur permettait pas d'être pris au sérieux en tant que rappeurs ${ }^{9}$, ce qui les pousse à nouveau à rompre leur contrat.

En 2013, Faada Freddy commence sa collaboration en solo au sein du label françaisThinkZik !, récemment créé par le franco-sénégalais Malick Ndiaye, neveu du musicien et compositeur sénégalais Wasis Diop. Faada Freddy y produit un nouveau projet, Untitled, dont le concept est de faire interpréter tous les instruments par des voix humaines et des percussions corporelles. Une année plus tard, alors que je débute ma recherche, c'est par hasard que je croise Ndongo D au Sénégal, après avoir tenté plusieurs fois de joindre les membres du groupe, qui se rendent très fréquemment à l'étranger. II me donne rendez-vous dans le studio de leur label indépendant Bois sakré, où je le retrouve à travailler sur les chansons de leur prochain album Foundations, qui sortira en 2016. Lors de cette séance, il me fait notamment écouter la chanson "Senegal ", qu'il me présente comme étant destinée à la diaspora sénégalaise résidant à l'étranger. Je lui fais remarquer que le groupe s'est exprimé à de nombreuses reprises sur le sujet de la migration dans ses chansons. Ndongo $D$ me répond que $c^{\prime}$ est un thème que le groupe connaît pour l'avoir expérimenté. Dans la chanson "Exodus " de l'album Boomerang, dédiée à " tous les clandestins, tous les bana bana (noms donnés aux vendeurs de rues sénégalais) qui cherchent l'eldorado en Americana ", les artistes se faisaient l'écho de représentations de l'ailleurs comme "Eldorado " et comme "Terre promise " où les migrants partaient dans l'espoir d'une vie meilleure et dont ils promettaient revenir avec "les poches pleines d'or ".

En revanche, I'intentionnalité première de la chanson "Senegal " est d'encourager les migrants à revenir au Sénégal, en suscitant la nostalgie du pays, mais aussi en évoquant une issue heureuse à l'expérience migratoire. Le titre peut aussi être compris comme une démonstration de l'amour des artistes pour leur pays ("j'aime ce pays qui m'a vu grandir ") ou encore comme une campagne de promotion touristique à destination d'un public étranger ${ }^{10}$.

Dans "Senegal ", les deux membres de Daara J Family expriment surtout des sentiments de nostalgie d'être "loin de chez soi " et de ses proches. Le pays est associé à une maison, à un " abri " vers lequel le migrant est appelé à rentrer tandis que la migration est présentée comme un déracinement. Le retour est vécu comme une consécration vers lequel aspire naturellement le migrant. La vie à l'étranger est alors conçue comme une parenthèse qui n'a qu'un seul but, celui de "préparer le chemin du retour". Les souffrances vécues à l'étranger sont ainsi rendues acceptables par la promesse de rentrer en champion (Banégas et Warnier, 2001).

9 Carnet de terrain (2014) Visite à Ndongo D (Daara J Family), Grand-Yoff/Dakar, Sénégal, 10/11/2014.

10 Le clip Sénégal a été réalisé en collaboration avec l'Agence Sénégalaise de Promotion Touristique. 
Dans " Senegal ", Ndongo D s'adresse au migrant en wolof :

"T'es loin de chez toi, rêves qu'on t'accueille un jour tu vas rentrer, prépares le chemin du retour saches que tu es proche de ton but, la famille, tout le monde t'attend, t'étais parti mais tu es revenu, le Sénégal est ton abri " (Daara J Family, 2016, traduction du wolof au français par El Hassan Diouf, journaliste à Rapdjolof.com)

Faada Freddy lui répond : " me revoilà chez moi, là où je me sens comme un Roi ».

En mettant en perspective l'année de sortie de ces chansons avec les étapes de la carrière artistique de Daara $\mathrm{J}$ puis de Daara $\mathrm{J}$ Family, les deux chansons acquièrent un nouveau sens. Dans la chanson "Exodus", diffusée en 2003 alors que le groupe est sous contrat avec une maison de disques qui leur fait espérer l'accession à une notoriété internationale, les membres du groupe croient en leur réussite par la migration, comme les "clandestins " auxquels la chanson est dédiée. En revanche, la chanson "Senegal ", diffusée dans le cadre d'un album qui se présente comme un retour aux sources, aux "fondations " sénégalaises du groupe, et qui est produit de façon indépendante, les paroles de la chanson mettent en relief, à travers le thème du retour de migration, le retour de Daara J Family au Sénégal. La chanson "Senegal " est alors symbolique de la carrière des membres du groupe qui, après des années de carrière internationale, expriment leur attachement à un pays d'origine qu'ils n'ont jamais vraiment quitté. Comme Ndongo D me le confiait en 2014, il est important pour les membres de retourner régulièrement au Sénégal, généralement tous les six mois. Cette résonance de l'imaginaire migratoire avec les mises en scène de I'identité artistique est particulièrement mise en exergue lorsque Faada Freddy chante que "le soldat n'a pas fui, mais qu'il est parti chercher de la force ". Tout comme il s'agit de ne pas accuser le soldat de désertion, puisqu'il n'a fui que pour revenir plus fort, il s'agit de ne pas accuser les migrants, et le groupe lui-même par la même occasion, d'avoir abandonné leur pays, puisqu'ils ne l'ont fait que pour revenir plus forts eux aussi.

Début 2018, Faada Freddy rentre au Sénégal, après une série de concerts donnés en Europe pour promouvoir son album solo, et y donner deux concerts, présentés dans la presse nationale comme clôturant triomphalement la tournée internationale de l'artiste. Interrogé par Radio France International, Faada Freddy s'exprime sur son retour au Sénégal : "C'est comme renaître. À chaque fois que je viens ici, je me ressource. À chaque fois que je reviens au Sénégal, j'ai l'impression que c'est la première fois "(Thibault, 2018). Dans la carrière du groupe Daara J Family, puis celle en solo de Faada Freddy, la mythologie du retour comme accomplissement est ainsi régulièrement réactivée au profit du prestige du groupe. Par la migration, " on fait grandir son nom en s'auréolant de la valeur-prestige rattachée à l'Ailleurs et à ses accessoires et en devenant ainsi l'incarnation de l'Illusion " (Fouquet, 2007). II s'agit alors de " partir pour grandir, revenir pour bâtir ", comme le proclame le groupe en conclusion du titre "Senegal ". 


\section{Wagëblë, une migration sans retour ?}

Wagëblë est un groupe formé par les rappeurs Waterflow et Eyewitness. Les deux membres se sont connus au lycée en 1994 et ont formé un premier groupe avec deux autres rappeurs avant que ces derniers ne partent à l'étranger. Wagëblë, qui signifie " du ghetto " (" wa " signifiant " du groupe de " et "gëblë " pour " ghetto ") est aussi I'acronyme de Wax Aduna Geddu Ettu Baatin Lemu Ëlek, qui signifie, en wolof, le lieu où les gens se réunissent pour parler des problèmes rencontrés. Comme leur nom le laisse supposer, le groupe s'inscrit dans la mouvance " hardcore " en utilisant le rap comme un instrument pour faire entendre "la voix des sans-voix ${ }^{11}$ ".

Les membres du groupe, originaires de Thiaroye, se présentent comme l'un des premiers groupes issus de "banlieue " et insistent sur la difficulté à se faire une place au sein d'une scène musicale alors dominée par des groupes issus du centre-ville dakarois. Selon Eyewitness, rencontré à Lausanne où il vit, c'est durant des auditions que le groupe se fait remarquer par le producteur norvégien Tom Roger du label Two Thou Entertainment au début des années 2000, alors même que le propriétaire du studio émet quelques réserves quant à leur laisser la possibilité de passer les auditions. Cette version des débuts du groupe, qui tranche avec d'autres versions, vise à mettre en lumière leurs difficultés à être reconnus, comme pour mieux justifier la distance que le rappeur a prise vis-à-vis d'autres artistes de rap au Sénégal, vers lesquels il s'est montré critique tout au long de notre rencontre.

Quoi qu'il en soit, le premier album du groupe, enregistré à Dakar avec Tom Roger, sort en 2003 et connaît un immense succès. Leur deuxième album, Sénégal, toujours enregistré par Two Thou Entertainment, est enregistré en Norvège et sort en 2005. Le groupe part en tournée internationale (États-Unis, Norvège, Suisse, Suède, France, Gambie, Guinée). Entre 2002 et 2005, pendant la période d'enregistrement de leurs deux albums et les tournées réalisées à l'étranger, le groupe voyage beaucoup, en retournant au Sénégal tous les six mois. Selon Waterflow, c'est en 2005 que les membres du groupe quittent le Sénégal pour s'installer de façon plus durable à l'étranger. Si Eyewitness s'installe très vite en Suisse après un court passage en Norvège - lorsque je le rencontre en 2014 il me dit être en Suisse depuis dix ans -, Waterflow vit d'abord quelques mois en Suisse avec Eyewitness, puis en Norvège avec des retours fréquents au Sénégal, avant de s'installer aux États-Unis quelques années plus tard, avec sa femme américaine et leur enfant âgé de quelques mois. II devient manager dans un restaurant belge pour pourvoir aux besoins de sa famille. Malgré la distance géographique et leurs activités professionnelles, le groupe publie un troisième album, Message of Hope, en 2011 sur leur label indépendant, Nubian Spirit. Waterflow, rencontré aux États-Unis en 2015, m'explique cette rupture avec le label norvégien comme une manière d'acquérir une nouvelle autonomie. À partir de cet instant, le groupe prend en charge toutes les facettes de leur activité artistique : production, enregistrement,

11 Les rappeurs sénégalais dits " hardcore " construisent leur " engagement musical " comme le fait d'utiliser leur média, la musique, pour faire entendre les voix des populations qui ne seraient pas, selon eux, écoutées par les décideurs politiques et plus largement, seraient inaudibles dans le système mondialisé. 
mixage, mais aussi design des pochettes d'albums, management des concerts, logistique, réalisation des vidéos, développement d'une marque de vêtements. Selon le rappeur, le titre de l'album Message of Hope symbolise l'évolution du groupe comme incarnation d'un message d'espoir : celui d'avoir réussi par leurs propres moyens. Ce message c'est aussi la réussite d'une trajectoire de vie, partie " de rien, des endroits les plus pauvres " et d'avoir utilisé ce qu'ils avaient à disposition pour " être là où on est maintenant " (Entretien, Washington DC., 2015).

Cette réussite en migration est illustrée dans la chanson "African Dream " de I'album Message of Hope. Ce "rêve " n'est pas présenté que sous un jour positif puisque les deux rappeurs y partagent les sacrifices qu'ils ont dû réaliser et soulignent les difficultés pour continuer leur carrière artistique en migration. Le clip notamment, met en scène de façon explicite les tentations auxquelles le migrant est exposé pour gagner de l'argent. II débute sur une séquence, sans musique, au cours de laquelle deux individus sont filmés en train de discuter en bas d'un immeuble, tout en roulant des joints entre leurs mains. Le premier, probablement un recruteur pour la vente de drogues demande au second, un de ses nouveaux vendeurs, s'il a bien vendu la nuit passée. Le vendeur lui répond qu'il s'est fait beaucoup d'argent et qu'il adore ce travail. Un des membres de Wagëblë, passe à quelques mètres devant eux pour rejoindre son appartement. Les deux hommes le regardent et partagent à son propos :

Le recruteur : "When you see that nigga over there man, going back and forth, back and forth, I don't know what he do man. Do he do music man? Or something like that? "

Le vendeur : "Man, music man, they don't have any money, man "

Le recruteur: "Yeah?"

Le vendeur : "Leave it leave it (tchiip). Music?"

Le recruteur : "That's right man we got to do our own, we here to make some dough.

We don't do no music. "

Cette conversation laisse place à une autre séquence qui filme Waterflow dans sa salle de bain, en train de se regarder dans le miroir. La séquence qui suit présente Eyewitness, devant son ordinateur. II est au téléphone avec un membre de sa famille à qui il explique qu'il ne sait pas quand il va rentrer au Sénégal. II raccroche, puis regarde des photographies de son dernier séjour au Sénégal, et se met à écrire la chanson "African Dream ".

Dans leurs couplets respectifs, les deux rappeurs racontent leur envie de rentrer chez eux, mais aussi leur détermination à ne pas abandonner " la mission ". Le refrain, chanté par le musicien et chanteur sénégalais reconnu Ismaël Lo, proclame que " l'Afrique les attend ". Le groupe est filmé dans un studio de musique, puis des images les montrent au Sénégal. La fin du clip reprend la conversation du début, où les deux hommes discutent encore de Waterflow, en disant de lui qu'il n'a pas d'argent et qu'il va se faire déporter. La dernière image du clip montre les membres du groupe dos à la caméra, face à I'horizon, comme pour signifier la promesse de meilleurs lendemains.

La chanson remplit plusieurs objectifs pour le groupe : elle répond d'abord aux attentes de leur public, en expliquant les raisons pour lesquelles le groupe n'est pas encore rentré au Sénégal tout en leur signifiant que, malgré les diffi- 
cultés, le groupe continue à exister. La sortie de l'album était d'ailleurs censée s'accompagner d'une tournée au Sénégal, après leur participation au Festival mondial des arts nègres ${ }^{12}$ (Festman). Selon une interview réalisée par les membres du groupe à cette occasion (Senerap, 2011), le groupe aurait préféré reporter la tournée, qui n'a finalement pas eu lieu, pour des problèmes d'organisation. Leur prestation lors du Festman a aussi été révélatrice de leur perte de popularité auprès d'un public rap qui n'a pas connu le groupe avant son départ à l'étranger. Pour le groupe, ce retour se présente comme un échec, qui renforce la distance prise par le groupe vis-à-vis de leur pays.

Interrogé sur sa relation avec le public sénégalais lors de notre rencontre aux États-Unis en 2015, Waterflow me confie ne pas chercher à atteindre un public spécifique ou à correspondre aux caractéristiques d'une musique définie comme du "rap sénégalais ", démarche décrite comme une fermeture :

"Si tu es sénégalais, tu te dis je fais du rap sénégalais, un rap qui est juste concentré sur la culture sénégalaise, qui parle que des problèmes des Sénégalais. Donc voilà, tu te fermes. Alors que le rap, dans son essence, $c^{\prime}$ est une culture universelle. $"^{13}$

Pour autant, Waterflow se définit toujours comme un rappeur sénégalais : son origine, " c'est dans mon DNA (ADN en anglais), mais ça détermine pas où je vais vivre toute ma vie ${ }^{14}{ }^{4}$ me dit-il.

En disant que son origine ne détermine pas son lieu de vie, il induit une capacité de chaque individu à ne pas être contraint par les circonstances, mais à choisir son propre destin, en d'autres mots " à se développer soi-même ", termes qu'il utilise pour motiver son envie de partir du Sénégal, rejoignant un imaginaire de la migration comme moyen de s'extirper des valeurs sociales qui empêchent l'individu de réussir (Mbodji, 2008). Chez Waterflow, ces valeurs sociales sont celles qui sont imposées aux artistes pour correspondre aux attentes du public sénégalais. Cet imaginaire migratoire se fond aussi dans le mythe américain du self-made-man qui se réalise par le rap à partir de rien. Cependant, tout comme l'artiste de rap qui a réussi doit rester "vrai " (Sköld, 2007), Waterflow, en tant que migrant, dit être resté le même et se revendique, malgré la distance, toujours comme " la voix des sans-voix".

C'est finalement vers la fin de l'année 2016 que le groupe retourne au Sénégal pour la cérémonie de remise des prix des Galsen hip-hop Awards, avant la sortie de leur nouvel album qui devrait s'appeler Witness the Flow (" sois témoin du flow ", jeu de mots sur leurs noms d'artistes). Sur scène, le groupe reprend ses chansons les plus connues, issues de leurs deux premiers albums, pour tenter de se reconnecter avec le public sénégalais. Le prochain album du groupe, qui n'est pas encore sorti, comporte notamment deux singles, "Jawale " et " Jambaar " qui sont sortis respectivement en aout 2016 et en octobre 2017. Dans le clip de « Jawale ", les membres du groupe se filment en train de marcher

12 Le festival mondial des arts nègres de 2010, organisée sous la présidence d'Abdoulaye Wade sur le thème de la renaissance africaine, est la troisième édition du festival, après les éditions de 1966, organisée par Léopold Sedar Senghor à Dakar, et celle de 1977, organisée à Lagos (Nigéria).

13 Waterflow (Wagëblë) (2015) Entretien, Washington, États-Unis, 01/10/2015.

14 Ibid. 
sur le Hollywood walk of fame, ce trottoir sur lequel sont apposées les 2600 étoiles de célébrités mondialement connues. Dans le clip de "Jambaar " en revanche, les membres du groupe se filment face à la mer, depuis les plages de Ngor, à l'extrémité nord-ouest de la ville de Dakar, ainsi que sur l'esplanade du Monument de la Renaissance africaine ${ }^{15}$. Cette production artistique sous-tend ainsi I'oscillation de Wagëblë entre le Sénégal et les États-Unis, entre l'image du migrant qui a réussi à l'étranger et retour au pays.

Comme chez Daara J Family, le refrain du titre "jambaar " proclame que "le guerrier est parti chercher de la force ". Chez Wagëblë, la migration est aussi expliquée par la recherche de meilleures perspectives ailleurs. Cependant, contrairement au premier groupe, l'expérience migratoire de Wagëblë ne s'est pas encore concrétisée par un retour définitif au Sénégal, ce dont rend compte les couplets des deux rappeurs. Waterflow y rappe notamment que le " vrai " soldat " travaille dur " et "s'envole ", faisant ainsi preuve de sa détermination à réussir et à triompher de toutes les difficultés, malgré les critiques :

"Réussir ou mourir j'emmerde mes ennemis Bosser dur et s'occuper de mon business Fais le tour du monde je me vante si j'en ai envie Je suis impoli et assume

T'as pas bien compris, il n'y a que Dieu C'est lui qui me donne du courage Je n'ai rien à craindre " (Wagëblë, 2017, traduction du wolof au français par El Hassan Diouf, journaliste à Rapdjolof.com)

\section{La figure du " jambaar ", entre imaginaire migratoire et positionnement artistique}

Les représentations de la migration chez les deux groupes de rap envisagés font appel à des éléments de l'imaginaire migratoire. Dans ces chansons, ces artistes motivent la migration par une recherche de meilleurs lendemains qui doit se solder par un retour dans le pays d'origine. Cependant, les paroles de ces chansons permettent aussi, de façon implicite, de lire la carrière artistique des artistes en rapport à une réussite internationale. L'emphase mise par les deux groupes sur la figure du jambaar (" guerrier " en wolof) est aussi notable, notamment vis-à-vis de l'absence du registre lié à I' " aventure ", que de nombreuses recherches ont relevée dans l'imaginaire migratoire en Afrique subsaharienne, notamment dans la littérature.

Dans Les Mots du Patrimoine, la figure de l'" aventurier " apparaît sous la forme de l'expression " partir à l'aventure " qui désigne le fait d' " aller tenter sa chance, chercher fortune dans une autre ville ou à l'étranger ". Selon Mazauric (2012 : 58), ce n'est pas un hasard si les récits de fiction privilégient la dimension aventureuse, puisque l'aventurier a une forte dimension romanesque. II est

15 Le monument est une statue de cinquante-deux mètres de hauteur représentant un homme africain enlaçant une femme et portant un enfant dans son bras gauche. L'enfant pointe son doigt vers ce qui, au-delà de I'horizon, mène aux côtes américaines, symbolisant une Afrique qui renaît en se dressant vers le ciel et vers l'ouest. 
celui qui " a rompu avec le cours des jours ordinaires, qui a choisi une autre vie, plus fertile en péripéties, en risques, voire en dangers, que le banal quotidien " et échappe au statut de victime pour être le maître de son propre destin. L'expression aurait été utilisée dès le milieu du XXe siècle pour désigner les voyages entrepris par de jeunes ouest-africains afin d'acquérir l'expérience nécessaire au passage à l'âge adulte. Pian (2009 : 11-12) définit " les aventuriers " comme ceux " désireux de partir à la découverte du monde et cherchant à s'émanciper des contraintes familiales et communautaires ".

Cependant, tous les migrants d'Afrique subsaharienne n'utilisent pas la figure de I'" aventurier " pour se décrire eux-mêmes, cette figure agissant même parfois comme repoussoir, en désignant des individus peu scrupuleux, qui recherchent une réussite rapide à tout prix. Chez Bredeloup (2008 : 289) par exemple, les " aventuriers " sont des trafiquants de diamants pour qui " la réalisation de soi prime sur le projet économique " en accord avec de nouveaux modèles de réussite correspondant à une " nouvelle économie morale imprégnant les imaginaires de l'État qui valorise et légitime les parcours fulgurants, les combines et les escroqueries, autrement dit tous les registres hétérodoxes de promotion individuelle quels qu'ils soient " (Ibid. : 291). À l'opposé, Havard (2001) soulignait l'émergence d'un " éthos Bul Faale " au Sénégal, promu par des rappeurs et des lutteurs, comme un modèle de réussite basé sur une éthique de l'effort et du travail. Ainsi, I'" aventurier ", comme celui qui part chercher fortune ailleurs par tous les moyens, et le membre du Bul Faale, qui grâce à ses efforts parvient à surmonter les obstacles posés par la société, se présentent-ils comme deux figures de réussite a priori contradictoires.

Parmi les nombreuses chansons de rap sénégalaises portant sur la migration, très rares sont les mentions faites à $I^{\prime}$ " aventure ". En revanche, un vocabulaire guerrier est très présent au travers de références au " combat " ou à la " mission " ainsi qu'à la " guerre " et à la " bataille ". Les figures utilisées renvoient au " guerrier ", mais aussi au " militaire ", au "tirailleur " et au " soldat " ainsi qu'aux " ennemis ". II s'agit de " contrer ", " combattre ", " brutaliser ", " anéantir ", " crier ", " envahir ", " tuer ", " calciner ", avec " force ", " courage ", " détermination ", " soif de vaincre ". Finalement, le guerrier a des " armes ", est "blindé comme un tank " et donne des " coups ". Cette rhétorique guerrière n'est en soi pas surprenante. Bazin (2001) soulignait déjà comment l'artiste de rap s'inspirait de l'imagerie guerrière et considérait le rap comme une arme, une métaphore souvent rattachée à la naissance du rap aux États-Unis. Le slogan "Love, peace and Having fun " du mouvement de la Zulu Nation illustre la manière dont la pratique du hip-hop se présentait comme une alternative à la violence des gangs : plutôt que de pratiquer la violence physique, les membres des gangs s'affronteraient autour de batailles de danse, de djing et de rap. Au Sénégal, la métaphore guerrière est importante dans le rap dit hardcore et son association avec des conditions de vie précaires. Le rappeur hardcore est " engagé et conscient ", mais par extension c'est aussi un " dur à cuire " ou autrement dit, un "Jambaar " (Moulard-Kouka, 2008). D'autre part, la figure du " soldat " est citée par quelques travaux sur l'imaginaire migratoire. Ainsi, si Bredeloup (2008) insiste sur la figure de l'" aventurier ", perceptible à travers le vocabulaire de la débrouille, l'astuce ou la chance, elle souligne que I'aventure renvoie aussi à une perspective guerrière, car le migrant doit être amené à surmonter des épreuves grâce à son courage, sa témérité, sa bravoure 
et sa fierté. Dans un texte diffusé en 2018, Mourre montre comment la figure du tirailleur est mobilisée dans la fabrication de l'imaginaire migratoire, notamment au travers de la mémoire collective de la " dette de sang ". Le sacrifice des tirailleurs durant la Deuxième Guerre mondiale est vengé par des migrants qui viennent réclamer cette dette, un thème que l'on trouve dans de nombreuses chansons de rap sénégalaises. À la lumière des carrières artistiques des artistes envisagés, ainsi que des éléments avancés précédemment au sujet de l'accusation de trahison des artistes qui partent de la part des artistes restés au Sénégal, je défends néanmoins que la figure du guerrier peut être analysée comme une alternative à celle de I'" aventurier ".

D'abord, contrairement à I'" aventurier ", qui part "tenter sa chance " et " découvrir le monde ", avant tout guidé par sa quête d'exotisme et son désir d'argent, le " soldat " a une " mission " : il ne fuit pas son pays, il part simplement ailleurs pour " chercher de la force " et ramener cette force au Sénégal. Ensuite, contrairement à l'" aventurier ", dont la réussite n'a pas de prix, le soldat conserve un sens du devoir et de la dignité (jom en wolof). Dans le clip " african dream ", la cinématographie a pour objectif de mettre en scène les efforts entrepris par les membres de Wagëble pour ne pas se détourner de leur " mission " première, faire évoluer leur musique, face à la tentation d'activités illégales plus rémunératrices comme la vente de drogue. La "guerre " que mène le rappeur est ici une guerre interne, contre ses propres impulsions de se détourner du "bon chemin ". Cette guerre interne peut se rapprocher de la notion de jihad telle que développée par I'Islam soufi, et notamment par Cheikh Ahmadou Bamba, chef religieux sénégalais fondateur de la confrérie mouride (Babou 2007). Selon Lamchichi (2005: 29), pour le soufisme, "le combat le plus important se situe au niveau intérieur, au niveau de l'ascèse, du jihad majeur (moral ou spirituel) que doit livrer le croyant d'abord contre lui-même, "à l'aide de son cœur", pour expulser de soi les pulsions négatives ". Contrairement à l' " aventurier ", le " soldat " ne sacrifie pas sa dignité au nom de la réussite. Pour reprendre l'expression utilisée par Maxi Krezy, le rappeur doit montrer qu'il n'est pas parti " torcher le cul des enfants blancs " et par conséquent, qu'il est encore digne de tenir le micro.

En se présentant comme des soldats en migration, ces artistes affirment ainsi ne pas avoir abandonné leur " mission " en tant que rappeurs et récusent les soupçons d'activités illégales associés à leur migration, leur permettant alors de négocier la continuité de leur appartenance au rap sénégalais, malgré leur expérience migratoire.

\section{Conclusion}

Cet article s'est attaché à aborder les imaginaires migratoires présents dans des chansons de rap sénégalaises, par leur mise en perspective avec les carrières artistiques de leurs interprètes, eux-mêmes insérés dans des trajectoires de mobilité. La création musicale n'est alors pas uniquement révélatrice d'imaginaires sur la migration, et le rap une représentation fidèle de la parole migrante. L'imaginaire migratoire doit en effet être relu à travers les processus de création musicale, qui impliquent un ensemble d'acteurs partageant un certain nombre de conventions. Par conséquent, chaque genre musical peut aussi être le lieu 
d'un renouvellement des significations apposés à ces imaginaires migratoires. Au Sénégal, les rappeurs se sont ainsi largement positionnés contre la migration " clandestine ", en déconstruisant les fantasmes sur l'Europe, et ont, en quelque sorte, cherché à contrecarrer un imaginaire migratoire existant, notamment en dénonçant l'idée de migration comme seul moyen d'accomplissement social, occultant dans ce sillage les intenses désirs de migrer et les rapports ambivalents entretenus vis-à-vis d'un Occident à la fois critiqué et admiré par les artistes de rap sénégalais. L'acte de rester au Sénégal fonde l'accomplissement de la " mission " d'un rappeur qui se doit de lutter pour l'avenir de son pays, malgré le rôle crucial joué par les mobilités dans les carrières d'un nombre croissant d'artistes. L'exploration de ces imaginaires à l'aune des carrières de leurs interprètes éclaire ainsi des processus d'inclusion et d'exclusion au sein des processus de création musicale : pour ceux dont l'expérience migratoire, et le retour, ne se justifient pas par la réussite, l'expérience migratoire devient une " source d'altérisation et d'exclusion entre de supposés "vrais" et de supposés "faux" " (Timera 2014 : 47). L'utilisation de la figure du " guerrier ", en se présentant comme une alternative à celle, décriée, de l' " aventurier ", devient alors une manière de détourner ces processus d'inclusion et d'exclusion, en revendiquant un attachement continu au rap sénégalais, malgré l'expérience migratoire.

\section{Références bibliographiques}

Avenel Cyprien (2009) La construction du " problème des banlieues " entre ségrégation et stigmatisation, Journal français de psychiatrie, 34, pp. 36-44.

Babou Cheikh Anta (2011) Le Jihad de l'âme. Ahmadou Bamba et la fondation de la Mouridiyya au Sénégal (1853-1913), Paris, Karthala Éditions, 366 p.

Banégas Richard et Warnier Jean-Pierre (2001) Nouvelles figures de la réussite et du pouvoir, Politique Africaine, 82, pp. 5-23.

Bazin Hughes (2001) La culture hip-hop, Paris, Desclée de Brouwer, 305 p.

Becker Howard (2008) Art Worlds, Berkeley, University of California Press, 410 p.

Becker Howard (1985) Les carrières dans un groupe professionnel déviant : les musiciens de danse, in Howard Becker, Outsiders : Études de sociologie de la déviance, Paris, Editions Metaillée, pp. 126-144.

Benga Ndiouga Adrien (2002) Dakar et ses tempos Significations et enjeux de la musique urbaine moderne (c.1960-années 1990), in Momar Coumba Diop Dir., Le Sénégal contemporain, Paris, Karthala, pp. 289-308.

Bredeloup Sophie (2008) L'aventurier, une figure de la migration africaine, Cahiers internationaux de sociologie, 125, pp. 281-306.

Canut Cécile et Alioune Sow (2014) Les voix de la migration. Discours, récits et productions artistiques, Cahiers d'études africaines, 213-214, pp. 9-25.

Charry Eric (2012) Hip Hop Africa: New African Music in a globalizing world, Bloomington, Indiana University Press, $404 \mathrm{p}$.

Clément Pierre-Alain (2015) La signification du politique dans le rap, Cultures \& Conflits, 97, pp. 123-141. 
Daara J (2003) Exodus, in Boomerang, BMG France, CD.

Daara J Family (2016) Senegal, in Foundations, Bois Sakré, [en ligne] consulté le 30/08/2015. URL : https://www.youtube.com/watch?v=JpvDC_s8aDg

Diouf Mamadou (2002) Les cultures urbaines entre traditions et mondialisation, in Momar Coumba Diop Dir., Le Sénégal contemporain, Paris, Karthala, pp. 261-288.

Fouquet Thomas (2007) Imaginaires migratoires et expériences multiples de I'altérité : une dialectique actuelle du proche et du lointain, Autrepart, 41 (1), pp. 83-98.

Forman Murray (2000) Represent: race, space and place in rap music, Popular Music, 19 (1), pp. 65-90.

Glick Schiller Nina and Noel B. Salazar (2013) Regimes of Mobility across the Globe, Journal of Ethnic and Migration Studies, 39 (2), pp. 183-200.

Guillard Séverin (2012) "Représenter sa ville " : I'ancrage des identités urbaines dans le rap des Twin Cities, Cybergeo : European Journal of Geography, [en ligne] consulté le 10/08/2018. URL : https://doi.org/10.4000/cybergeo.25357

Hammou Karim (2016) Rap et banlieue : crépuscule d'un mythe ?, Informations sociales, 190 , pp. 74-82.

Hammou Karim (2005) Comment le monde social du rap aménage-t-il son territoire ?, Sociétés contemporaines, 59-60 (3), pp. 179-97.

Havard Jean-François (2005) Bul faale ! : processus d'individualisation de la jeunesse et conditions d'émergence d'une "génération politique " au Sénégal, Thèse de sociologie politique, Université Lille 2, 729 p.

Herson Ben (2011) A Historical Analysis of Hip-Hop's Influence in Dakar from 1984-2000, American behavioral scientist, 55 (1), pp. 24-35.

Jeffries Michael P. (2011) Thug Life: Race, Gender, and the Meaning of Hip-Hop, Chicago, University of Chicago Press, 274 p.

Keyti (2003) Eldorado, in Jog ak Daanu, Cassette.

Kiwan Nadia and Meinhof Ulrike Hannah (2011) Music and Migration: A Transnational Approach, Music and Arts in Action, 3 (3), pp. 3-20.

Lamchichi Abderrahim (2005) Jihâd. Un combat contre quel adversaire ?, Mots. Les langages du politique, 1 (79), pp. 21-33.

Le Menestrel Sara (2012) Des vies en musique. Parcours d'artistes, mobilités, transformations, Paris, Éditions Hermann, $200 \mathrm{p}$.

Martin Denis Constant (2004) Les musiques en Afrique, révélateurs sociaux, Revue Projet, 283, [en ligne] consulté le 05/10/2017. URL : http://www.revueprojet.com/articles/2004-11-les-musiques-en-afrique-revelateurs-sociaux/

Martiniello Marco, Puig Nicolas et Gilles Suzanne (2009) Éditorial : Créations en migration, Parcours, déplacements, racinements, Revue Européenne des Migrations Internationales, 25 (2), pp. 7-11.

Maxi Krezy (2003) Départ volontaire, in Palanteeru Wallanteeru, Cassette. 
Mazauric Catherine (2012) Mobilités d'Afrique en Europe : récits et figures de l'aventure, Paris, Karthala, 383 p.

Mbodji Mamadou (2008) Imaginaires et migration : le cas du Sénégal, in MomarCoumba Diop, Le Sénégal des migrations : mobilités, identités et sociétés, Paris, Karthala, pp. 305-320.

Meyer Michaël, Adeline Perrot et Isabelle Zinn (2017) Entre ambition " tout-terrain " et impossible ubiquité : les ethnographes en mouvement, SociologieS, [en ligne] consulté le 04/01/2018. URL : http://journals.openedition.org/sociologies/6521

Milliot Virginie (2006) The "French Touch " : le hip-hop au filtre de I'universalisme républicain, Anthropologie et sociétés, 30 (2), pp. 175-97.

Moulard Sophie (2014) Le regard entre deux rives. La migration et l'exil dans le discours des rappeurs sénégalais, Cahiers d'Études Africaines, 213/214, [en ligne] consulté le 01/07/2014. URL : https://www.academia.edu/7500056/Le_regard_ entre_deux_rives._La_migration_et_lexil_dans_le_discours_des_rappeurs_senegalais_Cahiers_detudes_africaines_2014

Moulard-Kouka Sophie (2009) Le rap à Dakar. Mise en perspective du local et du global dans une culture populaire urbaine au Sénégal, in Yves Raibaud Éd., Comment la musique vient aux territoires, Pessac, Publications de la Maison des sciences de I'homme d'Aquitaine, pp. 153-163.

Moulard-Kouka Sophie (2008) "Senegal yewuleen! " Analyse anthropologique du rap à Dakar : liminarité, contestation et culture populaire, Thèse d'anthropologie, Université Victor Segalen-Bordeaux II, 575 p.

Mourre Martin (2018) Mémoire des tirailleurs et institutions des imaginaires migratoires au Sénégal dans les années 2000, Africa, 88 (3), [en ligne] consulté le 12/08/2018 URL : https://www.cambridge.org/core/journals/africa/article/africancolonial-soldiers-memories-and-imagining-migration-in-senegal-in-the-twentyfirst-century/57572DEDD31827E4DEAA5CB6A1CFDD8A\#

Navarro Cécile (2019) "Ce n'est pas le rap qui nous fait voyager, c'est nous qui faisons voyager le rap galsen ": Pratiques et Imaginaires $d^{\prime}($ im)mobilités au sein d'une scène musicale translocale au Sénégal, Thèse en sciences sociales, Université de Lausanne, $468 \mathrm{p}$.

Ndiaye Corréard-Geneviève (Dir.) (2006) Les Mots du Patrimoine. Le Sénégal, Paris, Éditions des Archives Contemporaines-AUF, 600 p.

Ortar Nathalie, Monika Salzbrunn et Mathis Stock (2018) Migrations, circulations, mobilités. Nouveaux enjeux épistémologiques et conceptuels à l'épreuve du terrain, Aix-en-Provence, Presses Universitaires de Provence, 230 p.

Pian Anaïk (2009) Aux nouvelles frontières de l'Europe : I'aventure incertaine des Sénégalais au Maroc, Paris, La Dispute, 237 p.

Rofheart Mahriana (2010) Don't abandon "our boat": shifting perceptions of emigration in contemporary Senegalese literature and song, Thèse en philosophie, Rutgers University/Graduate School, New Brunswick, 204 p.

Rose Tricia (1994) Black noise: Rap music and black culture in contemporary America, Hanover, NH, Wesleyan University Press, 237 p.

Salzbrunn Monika, Souiah Farida et Simon Mastrangelo (2015) Les " brûleurs " de frontières dans la musique populaire tunisienne, Afrique Contemporaine, 2 (254), pp. 37-56. 
Senerap (2011) Wagëblë: Exclusive Interview, Skyrock (blog), [en ligne] consulté le 21/02/2018. URL: https://senerap.skyrock.com/3032599760-WAGEBLE-EXCLUSIVE-INTERVIEW.html

Sheller Mimi and John Urry (2006) The New Mobilities Paradigm, Environment and Planning A, Economy and Space, 38 (2), pp. 207-226.

Sköld David (2007) Makin' It, by Keeping It Real. Street Talk, Rap Music, and the Forgotten Entrepreneurship from "the 'Hood", Group and Organization Management, 32 (1), pp. 50-78.

Souiah Farida (2011) Musique populaire et imaginaire migratoire en Algérie, Diversité, 164, pp. 27-33.

Thibault Guillaume (2018) Faada Freddy de retour au Sénégal, RFI Musique. fr, [en ligne] consulté le 16/04/2018. URL : http://musique.rfi.fr/musiqueafricaine/20180112-faada-freddy-retour-senegal

Timera Mahamet (2014) Mots et maux de la migration. De l'anathème aux éloges, Cahiers d'études africaines, 213-214, pp. 27-47.

Timera Mahamet (2001) Les migrations des jeunes Sahéliens : affirmation de soi et émancipation, Autrepart, 18, pp. 37-49.

Traïni Christophe (2008) La musique en colère, Paris, Les Presses de Sciences Po, $122 \mathrm{p}$.

Wagëblë (2017) Jambaar, Nubian Spirit, [en ligne] consulté le 09/10/2017. URL : https://www.youtube.com/watch?v=kfzl28pBd8g

Wagëblë (2016). Jawale, Nubian Spirit, [en ligne] consulté le 28/08/2016. URL : https://www.youtube.com/watch?v=LHnVLjw9ScQ

Wagëblë (2011) African Dream (Wagëblë Feat. Cheikh Lô), Message of Hope, [en ligne] consulté le 29/11/2014. URL : https://www.youtube.com/ watch?v=SLd4qm5_W_Q

Willems Roos (2008) Les "fous de la mer ": les migrants clandestins du Sénégal aux Îles Canaries en 2006, in Momar-Coumba Diop Éd., Le Sénégal des migrations : mobilités, identités et sociétés, Karthala Éditions, pp. 277-303. 


\section{Cécile Navarro}

\section{" Le soldat n'a pas fui, il est parti chercher de la force " : explorer les imaginaires migratoires à l'aune des carrières artistiques dans le rap au Sénégal}

De l'étude des imaginaires migratoires à l'étude des mobilités artistiques, la prise en compte de l'expression artistique a considérablement renouvelé I'approche des migrations. Les productions musicales ont notamment été considérées comme une manière privilégiée de recueillir les imaginaires migratoires. La musique, et notamment le rap, n'est-elle alors que la représentation fidèle d'une parole migrante ? Par la mise en perspective des imaginaires migratoires avec le vécu des mobilités au cours de carrières artistiques, à partir des exemples des groupes de rap sénégalais Wagëblë et Daara-J Family, I'article montre comment des artistes peuvent faire appel à un imaginaire migratoire particulier, celui du " soldat " plutôt que celui de l'" aventurier " pour mettre en scène leurs parcours artistiques, en fonction des conventions propres au genre musical auquel ils souhaitent appartenir.

\section{“The Soldier didn't Run Away, He Went Looking for Strength": Exploring Migration Imaginaries through Artistic Careers in Rap in Senegal}

From the study of migration imaginaries to the study of artistic mobilities, taking into account artistic expression has considerably renewed the approach of migrations. In particular, musical productions have been considered as a privileged way of collecting migration imaginaries. Is music, and especially rap, only then the faithful representation of a migrant voice? By putting migration imaginaries in perspective with the lived experiences of mobility during artistic careers, developing on the examples of Senegalese rap groups Wagëblë and Daara-J Family, the article shows how artists can appeal to a particular migration imaginary, that of the "soldier" rather than that of the "adventurer" to stage their artistic career, according to the conventions specific to the musical genre to which they wish to belong.

\section{"El soldado no escapo, se fue a buscar fuerza": explorar los imaginarios migratorios a través de las carreras artísticas en el rap en Senegal}

Del estudio de los imaginarios migratorios hasta el de las movilidades artísticas, tener en cuenta la expresión artística ha considerablemente renovado el enfoque de las migraciones. En particular, las producciones musicales han sido consideradas como una forma privilegiada de reunir imaginarios migratorios. ¿Es la música, y especialmente el rap, entonces solo la representación fiel de una palabra migrante? Al poner la imaginación migratoria en perspectiva con la experiencia de la movilidad durante carreras artísticas, utilizando los ejemplos de los grupos de rap senegaleses Wagëblë y Daara-J Family, el artículo muestra cómo los artistas pueden apelar a un imaginario migratorio particular, el de "soldado" preferablamente al de "aventurero", para poner en escena su carrera artística, de acuerdo con las convenciones específicas del género musical al que desean pertenecer. 